\title{
Das biopsychosoziale Modell
}

Josef W. Egger

Univ.-Prof., em. Professor für Biopsychosoziale Medizin, Medizinischen Universität Graz

\begin{abstract}
Die philosophische Frage nach dem «Menschenbild» der wissenschaftlichen Medizin ist zwar berechtigt, für den Patienten wichtiger ist allerdings, wie sehr ihm bei der Behandlung seines Leidens geholfen werden kann. Für einfach gelagerte krankhafte Vorgänge mag ein simples mechanistisches Verständnis wie das biomedizinische Krankheitsmodell ausreichen. Bei komplexeren Krankheitsprozessen kommt dieser Ansatz allerdings schnell an seine Grenzen. Besonders bei chronifizierten Störungen braucht es ein Verstehensmodell, das den Menschen nicht nur als komplex organisierte Materie oder als eine Aneinanderreihung von einzelnen Organsystemen begreift.
\end{abstract}

\section{Résumé}

La question philosophique de la "conception de l'homme" de la médecine scientifique est justifiée, mais pour le patient, l'essentiel est la mesure dans laquelle sa douleur peut être soulagée. Une compréhension mécaniste simple telle que le modèle biomédical peut suffire dans le cas de processus pathologiques basiques, mais il atteint rapidement ses limites avec les processus plus complexes. Les troubles chronicisés, en particulier, exigent un modèle de compréhension qui n'envisage pas seulement l'être humain comme de la matière à l'organisation sophistiquée ou une juxtaposition de systèmes organiques individuels.

Le modèle biopsychosocial de la médecine humaine peut ici servir de cadre théorique et s'entend dans sa version actuelle comme un modèle biopsychosocial étendu ou révisé, sous la forme d'une théorie de l'unité corpsâme.

La conception de l'être humain sous-jacente va au-delà de la vision jusqu'ici dominante, purement scientifique et biomédicale de I'homme. Elle abolit la dichotomie pathogenèse/salutogenèse (on peut être à la fois malade et en bonne santé) et considère qu'en plus du "guérisseur», le patient aussi a une obligation: les troubles complexes ne pourront être adéquatement soignés qu'au prix d'efforts conjoints et concertés. Cela ne diminue en rien l'importance de l'expert médical, mais cela valorise et émancipe le patient. À la fois à l'opposé des notions de «mécanisation de la médecine» ou «d'ingénierie biomédicale» (qui continueront de faire partie des activités médicales) et en même temps développement des actes médicaux en direction d'une meilleure coopération entre le médecin et le patient, la notion de «médecine narrative» s'est établie. La plupart des règlements d'études de médecine s'efforcent aujourd'hui d'améliorer la formation des prochaines générations de médecins à cet égard.
Unter dem Begriff Menschenbild (bzw. Menschenmodell) werden Annahmen über das Wesen des Menschen verstanden. Derartige Modelle leiten bewusst und unbewusst all unser forscherisches wie praktisches Handeln. Modelle vom Menschen sind auf einer Wertungsbasis, d.h. auf den Werten und Einstellungen der jeweiligen Gesellschaft gegründet und können streng genommen daher empirisch weder bestätigt noch widerlegt werden. D.h. sie sind einerseits von den gesellschaftlichen, philosophischen, kulturellen, ökonomischen sowie politischen Tendenzen der jeweiligen Zeit abhängig. Andererseits werden sie auch von den innerhalb einer wissenschaftlichen Gemeinschaft tradierten (bzw. sich durchsetzenden) Vorstellungen über den Menschen, dem zur jeweiligen Zeit vorherrschenden wissenschaftlichen Klima, von biographischen Momenten sowie vom Selbstbild der Theorie- bzw. Therapiegründer mitbestimmt. Sämtliche dieser Determinanten üben freilich einen wechselseitigen Einfluss aufeinander aus.

Menschenbilder als implizite Voraussetzungen für Theorien im Geltungsbereich der Humanmedizin sind natürlich für das ärztliche Handeln von weitreichender Bedeutung. Die wichtigste Funktion ist neben der heuristischen die konstituierende Funktion von Menschenmodellen. Mittels medizinischer Modelle, zumeist in Form von Metaphern, wird der Gegenstand der Medizin konstituiert und somit gleichzeitig Realität geschaffen, die sich im Sinne einer «self-fulfilling prophecy» fortwährend selbst bestätigt. Indem nämlich bestimmte Aspekte der Wirklichkeit beleuchtet wer- 
den, folgt daraus, dass sich auch die Forschungsmethoden der Medizin und die Praxeologie des ärztlichen Tuns (und damit das gesamte Handlungsspektrum medizinischer Interventionen) an diesen Gesichtspunkten orientieren. Daraus ergibt sich ein latentes Gefahrenmoment, dass wissenschaftliche Forschungim Besonderen auch im Bereich der Humanmedizin durch einen nicht ausreichend reflektierten mainstream auf bestimmte Aspekte der Wirklichkeit eingeengt wird. Dies behindert schon aus methodischen Gründen den Erkenntnisprozess und hat signifikante Folgen für das davon abgeleitete Handeln.

Im biomedizinischen Modell fehlt die Dimension des Menschen als denkendes und fühlendes Wesen.

Zumindest die letzten hundert Jahre dominiert im Bereich der wissenschaftlichen Heilkunde das sog. biomedizinische Modell. Es ist im Kern streng naturwissenschaftlich orientiert und basiert auf bahnbrechenden physikalischen und chemischen Erkenntnissen über den menschlichen Körper. Die westliche Welt ist zu Recht stolz auf diese Errungenschaften. Allerdings ist mit dem Triumph dieses Modells eine deutliche Verengung von potenziell weiteren, relevanten Dimensionen einhergegangen, sodass das dahinterliegende Menschenbild als «der Mensch als komplexe Maschine» kritisch beschrieben und diese Form der «modernen Medizin» salopp auch als «Reparaturmedizin» oder «Ingenieursmedizin» tituliert wurde. Tatsächlich fehlt im biomedizinischen Modell die Dimension des Menschen als denkendes und fühlendes bzw. als erlebendes und planerisch-handelndes Wesen, das immer in physiko-chemischen und sozio-kulturellen Umwelten eingebettet ist.

Die Schwachstelle des nach wie vor beherrschenden biomedizinischen Modells der Humanmedizin hat wohl auch damit zu tun, dass sich der öko-sozio-psychologische Wirklichkeitsbereich nicht oder nur rudimentär in das physiko-chemische Grundmodell integrieren liess. Seit Jahrhunderten wurden immer wieder theoretische Überlegungen angestellt, wie die Beziehung zwischen Körper und Seele (besser: zwischen Gehirn und Geist) zu erklären sei. Von all diesen Ansätzen ist - aus wissenschaftstheoretischen Gründen und auch aus Gründen der Kompatibilität mit dem naturwissenschaftlich-biomedizinischen Modell - bisher nur eines in der Lage, diese Problematik wenigstens einigermassen zu bewältigen: Das biopsychosoziale Modell ist hier das mit grossem Abstand meistgenutzte Paradigma [1]. Keine andere infrage kommende Theorie hat z.Zt. eine grössere Reichweite. Wie sich seit der ersten Ausformulierung vor ca. 40 Jahren inzwischen herausgestellt hat, ist dieser Ansatz für eine integrierte Medizin zwar wissenschaftlich äusserst fruchtbar und stellt alles, was die wissenschaftliche Medizin bis dato an Rahmentheorien zur Verfügung hatte, in den Schatten. Aber sie bereitet den potenziellen Nutzniessern doch einiges Kopfzerbrechen, weil das dahinterliegende Denkmodell ungeläufig und auch einigermassen komplex erscheint.

Es mag sein, dass ein wissenschaftstheoretisch so anspruchsvolles Konzept wie die Body Mind Unity Theory - sie beschreibt die aktuellste Version des biopsychosozialen Modells - für viele Gesundheitsexperten und insbesondere auch für Ärzte/-innen und Therapeut(inn)en eine Herausforderung für das Verstehen und Akzeptieren bedeutet. Aber dies ist kein redlicher Grund, auf simplere «Eigenlösungen» für das zu Recht kritisierte Mainstream-Modell der Medizin («der Mensch als komplexe Maschine», "Reparaturmedizin») auszuweichen, wie dies immer wieder versucht wird. Natürlich wird mit solchen begrenzten (idiosynkratischen) Modellen auch einem Bedürfnis breiter Schichten in der Bevölkerung nach möglichst einfachen Erklärungen Rechnung getragen - aber wissenschaftlich lauter ist das nicht. Es ist und bleibt die Bringschuld der aufgeklärten Experten, den Mitmenschen auch den weniger eingängigen Sachverhalt zugänglich zu machen. Denn die Wahrheit ist den Menschen zumutbar. So war es immer in der Menschheitsgeschichte, z.B. auch mit den Phänomenen der Götterwelt und Geister, des Magnetismus oder der Relativitätstheorie.

Mithilfe dieses Modells ist das «Maschinenmodell» der Medizin nicht bloss um die psychologischen, soziokulturellen und ökologischen Dimensionen erweitert worden im Sinne einer Aneinanderreihung dieser Wirkbereiche - selbst einer solchen Erweiterung begegnen die Vertreter des biomedizinischen Modells mit grosser Skepsis. Das Missverständnis bezüglich des biopsychosozialen Modells liegt schon in seinem von G.L. Engel etwas unglücklich gewählten Terminus, der suggeriert, dass es sich doch um eine Summenbildung bzw. summative Nutzung von biologischen + psychologischen + öko-sozialen Determinanten handeln könnte. Engel wollte allerdings mit dem Begriff seine vehemente Kritik an der vorherrschenden und gänzlich auf das Körperliche eingeengte «Reparaturmedizin» zum Ausdruck bringen und den Blick auf das komplexe Zusammenwirken von scheinbar subjektiven und objektiven Welten oder von «Lebensinwelten» und «Lebensumwelten» schärfen. Dies schafft er mit der Nutzung der sog. Allgemeinen Systemtheorie.

Tatsächlich handelt es sich dabei um ein evolutionäres 
Verständnis, wie sich Leben auf unserem Planeten bis zu den (höchst komplexen) psychologischen Phänomenen des Menschen weiterentwickelt hat. Erst mit Erarbeitung der "Allgemeinen Systemtheorie» - einer Metatheorie für das Verständnis, wie alles Lebende zusammenhängt - , welche im Kern auf L.v.Bertalanffy zurückgeht, wird mit dem Naturphänomen der «Emergenz» ersichtlich, dass es ab einem gewissen Grad der Komplexität zu Sprüngen in der Qualität von Lebenserscheinungen kommt. Zwar sind diese neuen Eigenschaften des Lebendigen vollkommen auf den jeweils darunterliegenden Elementen des Seienden aufgebaut, sie bilden aber einen eigenen Phänomenbereich mit jeweils typischen Eigenschaften. Eine Reduktion auf die darunterliegende Systemebene vermag das Typische dieser neuen Entität nicht ausreichend zu erklären, weil es auf dieser unteren Ebene diese Eigenschaften noch gar nicht gibt.

Für unsere Diskussion bedeutet dies: Seelische Phänomene (wie Fühlen, Denken, planerisches Handeln) sind auf der Ebene der neurobiologischen Gegebenheiten (der neurologischen Körperstruktur) weder adäquat erfassbar noch ausreichend zu verstehen, wenngleich sie immer auf der Basis dieser Vorgänge aufgebaut sind. - Solange die Allgemeine Systemtheorie (nicht zu verwechseln mit Kybernetik oder systemischen Algorhythmen) als Grundlage für das Verständnis des biopsychosozialen Modells nicht ausreichend verstanden ist, erübrigt sich jede Diskussion um dieses Modell. Aber selbst wenn man sich deren Implikationen bewusst ist, bleiben zumindest vorerst 2 fundamentale Problembereiche bestehen, wovon einer bisher gelöst werden konnte, für den anderen allerdings weiterhin eine Lösung aussteht.

Problem Nr.1: In Wechselwirkung können nur Entitäten (für sich genommene "echte» Strukturelemente oder Wirkelemente) der Welt stehen. Im Schema von Descartes wären dies Körper/Seele, auf dessen angenommener Wechselwirkung auch die herkömmliche Psychosomatik aufbaut. Dieser Ansatz ist allerdings längst als wissenschaftstheoretisch unlogisch erkannt und verworfen worden. Denn wo soll denn die «Seele» verortet sein (wo soll das Seelische «sitzen»), das als eigenständiges Phänomen in Wechselwirkung mit dem Körperlichen treten soll und die physikalische Entität des materiellen Körpers beeinflussen soll? Dazu müssten wir unsere Grundgesetzte der Welterklärung (physiko-chemische Grundgesetze) zertrümmern, wozu kein Anlass besteht.

Dieses Problem konnte mit der Integration der «LeibSeele-Identität» von Spinoza gelöst werden. Damit haben wir allerdings auch das ursprüngliche biopsychosoziale Modell, wie es von G.L. Engel in den späten
70er-Jahren des vorigen Jahrhunderts propagiert wurde, erheblich erweitert. Wir müssten ab sofort von einem revidierten oder erweiterten biopsychosozialen Modell sprechen oder besser von der "Theorie der Körper-Seele-Einheit» (body mind unity): Die Seele - oder besser: all das, was wir als seelische Phänomene bezeichnen - erwächst aus der Evolution. Sie ist das erkennbar komplexeste Phänomen der Entwicklungsgeschichte des Lebens. Alles Seelische ist damit nicht jenseits der Biologie angesiedelt oder verstehbar, sondern erwächst aus ihr. Im Sinne der Allgemeinen Systemtheorie bildet sie zugleich einen eigenen «Wirklichkeitsbereich", der sich allerdings nicht mehr auf die darunterliegenden (biologischen) Systemebenen reduzieren lässt.

Problem Nr. 2: Weil wir weiterhin zwei unterschiedliche Sprachsysteme zur Beschreibung des Körperlichen einerseits und Seelischen andererseits haben, bleibt die Schwachstelle bestehen, dass wir bis auf Weiteres über kein vereinheitlichtes Begriffssystem verfügen, in der die biologischen und psychologischen Phänomene als zusammengehörende Wirklichkeitsaspekte beschreibbar wären.

\section{Auf der Ebene der Materie können wir für} das sogenannte Seelische kein ausreichendes Verständnis erlangen.

Wie uns bereits A.N. Whitehead gelehrt hat, gilt: Die Materie ist nicht "tot», sondern als hochdynamisches Phänomen zu sehen, in dem alle komplexeren Phänomene (und somit auch das Leben) in seinen Grundbausteinen bereits enthalten sind. Der "Sternenstaub", aus dem alles besteht, was ist, kann unter hochkomplexen Bedingungen Organisches hervorbringen, wobei dieses Lebendige wiederum unter höchst aufwändigen evolutionären Prozessen auch seelische Phänomene generieren kann. Diese Phänomene sind aber wiederum von einer höheren Komplexität als die darunterliegenden materiellen Strukturen, sodass wir auf der Ebene der Materie für das sogenannte Seelische kein ausreichendes Verständnis erlangen können. Die Erkenntnis, dass der (fälschlicherweise als «tot» bezeichneten) Materie die prinzipielle, inhärente Möglichkeit zum Hervorbringen von Lebendigem und im Weiteren auch von Formen des Seelischen gegeben ist, ist allerdings aufgrund jahrtausendealter gegenläufiger Denktraditionen und sprachlichen Konventionen noch nicht weit verbreitet.

\footnotetext{
Literatur

1 Egger JW. Theorie und Praxis der biopsychosozialen Medizin. Körper-Seele-Einheit und sprechende Medizin. Wien: Facultas; 2017.
} 